\section{Salud, ambiente y desarrollo sostenible en México ${ }^{1}$}

1 Basado en el documento de trabajo "Salud, ambiente y desarrollo humano sostenible: el caso de México", elaborado en junio de 1997 por el Comité Técnico Nacional para el Desarrollo Sostenible, conformado con representantes de las diversas dependencias de la administración pública federal responsables de la salud pública, la gestión ambiental y las políticas de desarrollo, así como con representantes de la Organización Panamericana de la Salud, el Programa de las Naciones Unidas para el Medio Ambiente y el Programa de las Naciones Unidas para el Desarrollo.
En octubre de 1995 se llevó a cabo en Washington, DC, la Conferencia Panamericana sobre Salud y Ambiente en el Desarrollo Humano Sostenible, en donde se establecieron los principios de política y estrategia, las prioridades comunes y las responsabilidades compartidas que se proponen adoptar los países participantes. Como parte de los acuerdos emanados de la Conferencia, México se comprometió a elaborar un diagnóstico sobre los problemas de salud pública asociados con sus condiciones ambientales y su proceso de desarrollo. Para ello, a finales de 1995 se integró el Comité Técnico Nacional para el Desarrollo Sostenible, destinado a promover la generación de un proceso sostenido y de consenso para proponer áreas de intervención y proyectos concretos en este campo. Así, en este documento se presentan los hallazgos más importantes del Comité y sus recomendaciones básicas con respecto a la planeación en México en materia de salud, ambiente y desarrollo.

\section{PROBLEMAS Y PRIORIDADES}

El objeto primordial del desarrollo es mejorar las condiciones en que vive el ser humano. Sin embargo, el proceso de desarrollo puede menoscabar gravemente la salud, al alterar las condiciones tanto ambientales como socioeconómicas que afectan a la población. Como ejemplos cabe mencionar la contaminación industrial, el agotamiento de recursos naturales, el colapso de sistemas rurales de subsistencia, las condiciones de hacinamiento en el medio urbano y una creciente exposición de segmentos de la población a riesgos ambientales que inciden sobre la salud.

Para poder hacer frente a los problemas que acarrea el desarrollo, resulta indispensable contar con un diagnóstico adecuado de la importancia y jerarquía relativa de los problemas de salud ambiental predominantes en una sociedad. En el caso de México, ello se hizo estableciendo un perfil epidemiológico del país que sirviera de base para definir prioridades en la materia.

México tiene en la actualidad un perfil epidemiológico mixto, con características de transición prolongada y de polarización. La transición prolongada se refiere al cambio del patrón de mortalidad en los últimos 50 años. De cifras elevadas asociadas con las defunciones por padecimientos transmisibles se ha progresado hacia una mortalidad general e infantil bajas. Este cambio ha sido consecuencia 
de la urbanización, la ampliación de la cobertura de los servicios de salud, la industrialización y otros factores asociados con el desarrollo.

La polarización se refiere al hecho de que la transición no se ha dado en todos los sectores de la población en la misma magnitud o con el mismo ritmo. Así, persisten áreas rurales donde prevalece un patrón de mortalidad alto asociado con padecimientos infectocontagiosos, al igual que grupos sociales marginados de los beneficios del desarrollo.

Como resultado de los cambios económicos, del aumento de la industrialización y de la concentración de la población en áreas urbanas, el panorama de la morbilidad también se ha modificado. Entre estas modificaciones destacan la disminución de las enfermedades transmisibles, el aumento de las enfermedades no transmisibles y el aumento, aunque menos marcado, de las lesiones ocasionadas por accidentes y actos de violencia.

Los cambios en la estructura poblacional han hecho evidente que México se encuentra en una etapa de transición demográfica que ha sido originada, en parte, por el desarrollo económico y la industrialización iniciados en los años cuarenta. Entre los efectos más importantes de estos cambios se pueden señalar:

- Las tasas de mortalidad general y de menores de un año disminuyeron sostenidamente. La primera se redujo de 23 defunciones por 100000 habitantes en 1940, a 4,7 en 1994 y la segunda, de 158,7 a 29 defunciones por 1000 nacidos vivos registrados para el mismo período.

- La fecundidad disminuyó 59\% entre 1960 y 1994.

- La esperanza de vida aumentó de 40 años en 1940, a 72 en 1994.

- La población aumentó de 19,6 millones de habitantes en 1940 a 81,2 en 1990, lo cual es un reflejo de la disminución de la mortalidad más el aumento de la esperanza de vida.

- La tasa anual de crecimiento poblacional aumentó de 2,7\% en 1940 a 3,1\% entre 1960 y 1970, y disminuyó a 1,8\% en 1993.

- La concentración poblacional en áreas urbanas aumentó de 35\% en 1940 a $71 \%$ en 1990, lo que refleja la creciente industrialización.

La información anterior sirvió para identificar, partiendo de criterios básicos de jerarquización (cuadro 1), los problemas de salud ambiental prioritarios y secundarios (cuadro 2).

\section{ÁREAS DE INTERVENCIÓN}

El Comité Técnico Nacional también detectó los principales problemas de carácter estructural: falta de un sistema de información integrada en materia de salud, ambiente y desarrollo; falta de
CUADRO 1. Criterios básicos de jerarquización de problemas de salud ambiental

- La población afectada, tanto en su magnitud como en sus características sociodemográficas

- Las condiciones de salud detectadas en dichas poblaciones

- El grado de sensibilidad de los grupos poblacionales a los problemas ambientales

- Las posibilidades reales de emprender acciones interinstitucionales integradas y coordinadas

canales de comunicación inter e intrainstitucional y sectorial, y falta de coordinación para planear y ejecutar programas y acciones en este campo. Esto sirvió de base para establecer tres áreas de intervención directa, las cuales se presentan a continuación.

Sistemas de información: diseñar un sistema único de información sobre las condiciones ambientales y de salud. Esto permitiría tomar decisiones informadas para mantener, orientar o reorientar los programas relacionados con la salud y el medio ambiente.

Canales de comunicación: establecer canales formales y eficaces de comunicación entre los sectores involucrados en los problemas de salud ambiental (dependencias federales, gobiernos estatales y municipales, iniciativa privada, sector académico, organizaciones civiles y población en general).

Coordinación intersectorial: generar una instancia permanente de gestión intersectorial para aten-

CUADRO 2. Problemas de salud ambiental. México, 1997

\begin{tabular}{lc}
\hline \multicolumn{1}{c}{ Prioritarios } & Secundarios \\
\hline - Abastecimiento de agua & - Seguridad y calidad de los \\
potable & alimentos \\
- Disposición de aguas & - Uso de la tierra en zonas \\
servidas y excretas & urbanas y rurales \\
- Control de residuos & - Asentamientos humanos y \\
domésticos & vivienda \\
- Control de residuos & - Exposición a insectos y \\
peligrosos & otros vectores \\
- Calidad del aire en el hogar, & - Riesgos del ambiente en el \\
trabajo y ambiente exterior & trabajo \\
- Calidad de las aguas & - Control del ruido residencial, \\
superficiales, subterráneas & industrial y de vehículos \\
y costeras & - Control de la exposición a \\
- Control de los productos & radiaciones \\
químicos en el ambiente & - Efectos de los transportes \\
- Desastres naturales y & en el ambiente y la salud \\
accidentes industriales y & - Efectos de los viajes y \\
nucleares & turismo \\
\hline
\end{tabular}


der de manera específica a los problemas de salud y ambiente asociados con el proceso de desarrollo.

\section{RECOMENDACIONES}

- Diseñar y aplicar un programa para analizar, evaluar y actualizar el marco jurídico vigente relacionado con los temas de desarrollo sostenible.

- Establecer una instancia de coordinación intersectorial en materia de salud, ambiente y desarrollo, buscando diagnosticar los problemas sanitarios relacionados con el medio ambiente, determinar su importancia relativa, y planear y ejecutar acciones y programas para resolverlos.

- Establecer canales de comunicación intersectoriales para el manejo institucional de programas en salud ambiental.

- Evaluar la posibilidad de establecer un sistema de información que integre los hallazgos del Sistema de Información Epidemiológica con los del Sistema de Información Ambiental, a fin de evaluar los riesgos del ambiente para la salud y así respaldar la jerarquización de acciones en este campo.

- Reforzar los programas de investigación que realizan las instituciones de investigación, a fin de integrar sus resultados con la toma de decisiones en materia de salud y ambiente.

- Homogeneizar el sistema nacional de información sobre morbilidad; continuar mejorando la calidad de los datos de mortalidad, y establecer mecanismos que los correlacionen con información ambiental.
- Reforzar la utilización de la información sobre salud ambiental para mejorar el conocimiento de las relaciones entre factores ambientales y daños a la salud, con la finalidad de identificar puntos de intervención que permitan modificar los factores de riesgo.

\section{SYNOPSIS}

\section{Health, the environment, and sustainable development in Mexico}

This article is based on "Salud, ambiente y desarrollo humano sostenible: el caso de México," a document prepared in June 1997 by the Comité Técnico Nacional para el Desarrollo Sostenible. It opens with information regarding the epidemiologic and demographic changes that have taken place in Mexico, such as the decrease in communicable diseases, the rise in noncommunicable diseases, and the less conspicuous increase in lesions resulting from accidents or acts of violence. This is followed by a discussion of priority problems and problems of lesser magnitude in environmental health, specifically those relating to water and air quality, as well as disposal of household and dangerous wastes. Finally, it proposes three areas of intervention in light of the structural problems detected: the absence of an integrated information system covering the area of health, environment, and development; the absence of channels of communication within and between institutions and sectors, and the lack of coordination in planning and implementing programs and actions in this field. 